\title{
Tolerance of start-up control of rotation in parametric pendulum by delayed feedback
}

$\operatorname{AUTHOR}(S)$ :

Yokoi, Yuichi; Hikihara, Takashi

\section{CITATION:}

Yokoi, Yuichi ... [et al]. Tolerance of start-up control of rotation in parametric pendulum by delayed feedback. Physics Letters A 2011, 375(17): 1779-1783

\section{ISSUE DATE:}

2011-04

URL:

http://hdl.handle.net/2433/159447

\section{RIGHT:}

(c) 2011 Elsevier B.V.; This is not the published version. Please cite only the published version.; この論文は出版社版でありません。引用の際に は出版社版をご確認ご利用ください。 


\title{
Tolerance of start-up control of rotation in parametric pendulum by delayed feedback
}

\author{
Yuichi Yokoi*, Takashi Hikihara \\ Department of Electrical Engineering, Kyoto University, Nishikyo, Kyoto 615-8510, Japan
}

\begin{abstract}
In this paper, we propose a control method for establishing periodic rotation inherent in parametric pendulum based on a delayed feedback control. The experiments elucidate the existing range of periodic rotation in the domain of delay. The range of existence possibly represents the tolerance of proposed control with mistuned delay. It is confirmed that forced synchronization governs the existence and the width. The result assures that the frequency synchronization characteristics overcome the mistuned difference of delay in the control through entrainment.
\end{abstract}

Keywords: Delayed feedback control, Bifurcation diagram, Synchronization, Start-up control, Rotation, Parametric pendulum

\section{Introduction}

Pendulum is a simple physical system, but is of significance in science and engineering. A parametrically excited pendulum demonstrates coexisting oscillation and rotation. Rotation of parametric pendulum [1-3] exhibits a conversion from the external vibration into its rotational motion. The converting motion is effective from the viewpoint of applications such as energy scavenging from vibration of nature [4]. In this paper, we propose a control method for establishing the periodic rotation inherent in the parametric pendulum based on a delayed feedback control. The tolerance of the control method with mistuned delay is confirmed experimentally.

Delayed feedback control [5] is one of practical methods for controlling chaos [6-8]. It is well known that the control method is proposed for continuous systems based on the OGY method [6] to stabilize an unstable periodic orbit embedded in chaotic attractors. A lot of experimental applications of the control have been carried out with benefits of the property to require no exact system model [9-12]. On the implementation of the control, the setting of the delay is requested at the exact period of the target orbit for the stabilization of the unstable periodic orbit. Then the control gain is adjusted on an empirical, theoretical or numerical basis. The adjustment is comparatively easy because the delayed feedback control stabilizes the target orbit in a certain range of the control gain [5]. On the other hand, the period of target orbit has to be estimated correctly if we have no preliminary information of the period. The bifurcation diagram with respect to the delay time reveals that the delayed feedback control still settles the chaotic system into a periodic orbit in spite of the mistuned delay time [5, 12-14]. The range of periodic orbit in the diagram is called window. The result indicates the substantial adjustability on the delay.

\footnotetext{
${ }^{*}$ Corresponding author.

Email address: yokoi@dove.kuee.kyoto-u.ac.jp (Yuichi Yokoi)
}

In comparison with the control gain, the delay time has not attracted attentions in terms of the effect to the performance of control, which might be caused by the difficulty in functional analysis and calculation [15].

The delayed feedback control can substantially synchronize the current state of the system to its delayed state $[11,16]$. The control is associated with synchronization in chaotic systems [17]. The current state, the delayed state, and the feedback loop correspond to a response subsystem, a drive subsystem, and a coupling, respectively. Control and synchronization for chaos can be applied to periodic orbit. Based on the delayed feedback control, we propose a control method to establish stable periodic rotation depending on the initial condition and the external excitation. This paper focuses on experimental investigations for window of periodic rotation in the domain of delay. The existence and width of the window is governed by frequency synchronization.

The proposed control is implemented to an experimental setup of parametric pendulum for maintaining the periodic rotation. Since the periodic rotation coexists with low energy states and motions [18], the control is required to sustain the periodic rotation against irregularity, noise, and frequency variation of vibration of nature in the energy scavenging. In particular, the frequency variation causes the mistuned delay in the control scheme. It is also confirmed that the existence of the window of periodic rotation in the domain of delay possibly represents the tolerance of proposed control with mistuned delay. The tolerance with incorrect delay is definitely associated with the stability of delay systems [19].

\section{Experimental setup and start-up control}

The experimental setup shown in Fig. 1 for parametric pendulum is constructed by exciting a mechanical pendulum vertically. The mechanical pendulum is configured at the mass $m=$ $189.1 \mathrm{~g}$ and the length $l=138.3 \mathrm{~mm}$ by constructing a rod and 


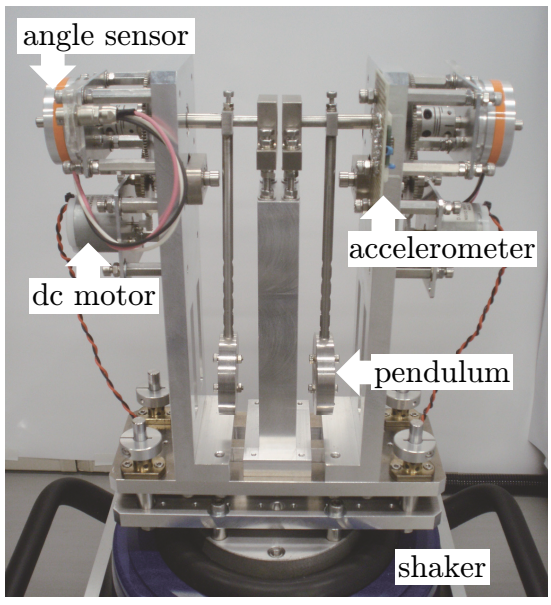

Figure 1: Experimental setup for parametric pendulum.

\begin{tabular}{llll}
\multicolumn{4}{c}{ Table 1: Size of mechanical pendulum. } \\
\hline Rod & \multicolumn{3}{c}{ Bob } \\
\hline Mass & $39.6 \mathrm{~g}$ & Mass & $144.9 \mathrm{~g}$ \\
Length & $180.0 \mathrm{~mm}$ & Diameter & $50.0 \mathrm{~mm}$ \\
Diameter & $6.0 \mathrm{~mm}$ & Width & $10.0 \mathrm{~mm}$ \\
\hline
\end{tabular}

a bob in Tab. 1. The pendulum is supported by a mechanical rig mounted on an electromagnetic shaker. The shaker generates a sinusoidal excitation in the vertical direction which corresponds to the parametric excitation. We propose a control method for establishing the periodic rotation inherent in the experimental setup. Fig. 2 shows the block diagram of the proposed control implemented to the vertically excited mechanical pendulum. The dynamics of the experimental setup is described by

$$
\begin{aligned}
& \frac{\mathrm{d} \theta}{\mathrm{d} t}=v, \\
& \frac{\mathrm{d} v}{\mathrm{~d} t}=-\frac{D(\theta, v)}{m l^{2}}-\frac{g+a \cos (2 \pi f t+\phi)}{l} \sin \theta+\frac{F u(t)}{m l^{2}}, \\
& u(t)=K(\theta(t-\tau)+\Theta-\theta(t)),
\end{aligned}
$$

where $t$ denotes the time, $\theta$ the angular displacement of pendulum from the downward position, $v$ the angular velocity, and

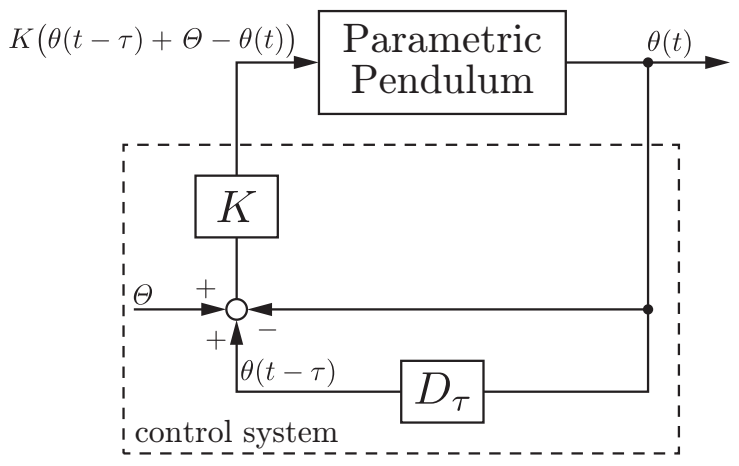

Figure 2: Block diagram of the start-up control with time delay for the periodic rotation inherent in the parametric pendulum.

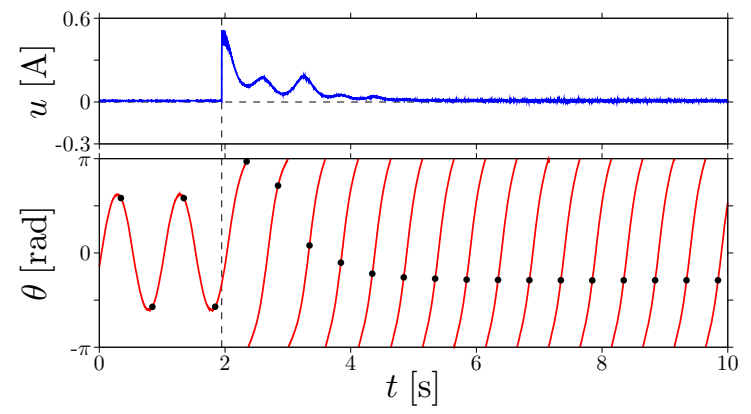

Figure 3: Establishment of the periodic rotation inherent in the vertically excited mechanical pendulum from its periodic oscillation at $a=1.1 \mathrm{~m} / \mathrm{s}^{2}$, $f=2 \mathrm{~Hz}, K=0.072 \mathrm{~A} / \mathrm{rad}, \tau=T=1 / f=0.5 \mathrm{~s}$, and $\Theta=2 \pi$. The points in the figure below denote the stroboscopic points taken at every excitation pe$\operatorname{riod} T$. The vertical dash line represents the moment of onset of the control.

$g$ the gravity acceleration. The vertical excitation is regulated with the amplitude $a$ and the frequency $f$. The constant $\phi$ denotes the initial phase of excitation. Since we have no exact model of the damping effect, the damping here is described as the function $D(\theta, v)$. The linear viscous coefficient is estimated at around $1 \times 10^{-4} \mathrm{~N} \cdot \mathrm{m} / \mathrm{s}$. The function $u(t)$ denotes the control input with the control gain $K$, the delay time $\tau$, and the periodicity on $\Theta$. The control input $u(t)$ is applied as a torque to the mechanical pendulum by a DC motor through gears with $F=0.18 \mathrm{~N} \cdot \mathrm{m} / \mathrm{A}$. The required angular displacement $\theta$ is measured by an angle sensor, which consists of angular potentiometer. The delayed feedback loop can be implemented as a program in a computer for control with $\mathrm{A} / \mathrm{D}$ and $\mathrm{D} / \mathrm{A}$ converters. Now we target the periodic rotation at which the pendulum rotates once during the excitation period $T=1 / f$. For the target rotation the angular displacement $\theta(t)$ exhibits the periodicity $\theta(t)=\theta(t-T)+2 \pi$. Thus we set the delay time $\tau=T$ and the periodicity on $\Theta=2 \pi$ so that the establishment of periodic rotation is accomplished. Here it should be noted that the control parameter $\Theta$ cannot be replaced by carrying out modulo $2 \pi$ operation for $\theta$. Consider a situation which can be described as $\theta(t)=3 \pi+\epsilon$ and $\theta(t-T)=\pi-\epsilon$ under the control, where $\epsilon$ is a small parameter. This represents that the pendulum rotates almost periodically. The modulo $2 \pi$ operation possibly changes from $\theta(t)=3 \pi+\epsilon$ to $\theta(t)=-\pi+\epsilon$. Then, under the start-up control without $\Theta$, the control input is obtained at $u(t)=2 K(\pi-\epsilon)$. The corresponding torque is too large to establish the periodic rotation even though the pendulum exhibited almost periodic rotation. Therefore, the control parameter $\Theta$ is necessary to reflect the periodicity of rotation. For plotting the following figures, we perform the modulo $2 \pi$ operation for the angular displacement $\theta(t)$.

Figure 3 shows an example of the control for starting up the periodic rotation inherent in the experimental setup. The vertical excitation is fixed at $a=1.1 \mathrm{~m} / \mathrm{s}^{2}$ and $f=2 \mathrm{~Hz}$ so that the periodic rotation coexists with a periodic oscillation. The control gain is adjusted at $K=0.072 \mathrm{~A} / \mathrm{rad}$. The result shows that the periodic rotation is established from the periodic oscillation. After the establishment of rotation, the control input $u(t)$ disappears. It suggests that the periodic rotation is inherent in 


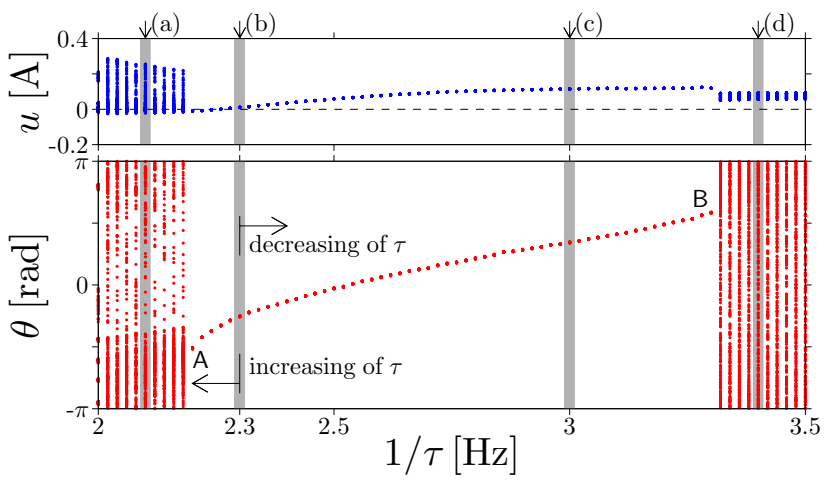

Figure 4: Bifurcation diagram of rotation with respect to the delay time $\tau$ in the experimental setup under the start-up control. The vertical excitation is fixed at $a=1.2 \mathrm{~m} / \mathrm{s}^{2}$ and frequency $f=2.3 \mathrm{~Hz}$. The control parameters are adjusted at $K=0.072 \mathrm{~A} / \mathrm{rad}$ and $\Theta=2 \pi$. The diagram is plotted by upward and downward shift of the control frequency $f_{\tau}$ corresponding to the reciprocal of the delay time $\tau$ from the excitation frequency $f=2.3 \mathrm{~Hz}$. The points denote the stroboscopic points of steady rotations.

the experimental setup at the parameters.

\section{Bifurcation with respect to delay}

Figure 4 shows an experimental bifurcation diagram of rotation with respect to the delay time $\tau$ at $a=1.2 \mathrm{~m} / \mathrm{s}^{2}, f=2.3 \mathrm{~Hz}$, $K=0.072 \mathrm{~A} / \mathrm{rad}$, and $\Theta=2 \pi$. The diagram is plotted through the stroboscopic observation at every excitation pe$\operatorname{riod} T=1 / f=1 / 2.3 \mathrm{~s}$. The points represent steady rotations measured by decreasing and increasing the delay time $\tau$ from the excitation period $T$. According to the experimental procedure, we display the bifurcation parameter at the reciprocal of the delay time, denoted by $1 / \tau=: f_{\tau}$, in Fig. 4 .

The inherent periodic rotation is maintained by fixing the delay time at $\tau=T$ in the delayed feedback loop. Fig. 5(b) shows the periodic rotation with the null control input $u(t)$. The periodic rotation is denoted by the single stroboscopic point in Fig. 4, which implies that the period of the rotation is coincident with the excitation period $T$. Decreasing the delay time $\tau$ shifts the stroboscopic point of angular displacement $\theta(t)$ in the positive direction. The shifted single stroboscopic point corresponds to a periodic rotation that does not exist without the control. We show the controlled periodic rotation at $\tau=1 / 3 \mathrm{~s}$ in Fig. 5(c). The control input $u(t)$ remains and vibrates periodically. Further decrease of the delay time $\tau$ induces a bifurcation marked by $B$ in Fig. 4. At around $\tau=1 / 3.3 \mathrm{~s}$ the periodic rotation disappears and a quasiperiodic rotation appears. The quasiperiodic rotation is depicted by a number of the stroboscopic points in Fig. 4. Fig. 5(d) shows the quasiperiodic rotation at $\tau=1 / 3.4 \mathrm{~s}$. The average frequency is higher than the excitation frequency. Increase of the delay time $\tau$ shifts the the stroboscopic point of $\theta(t)$ in the negative direction in a symmetric fashion. By increasing the delay time $\tau$ to $1 / 2.18 \mathrm{~s}$, another bifurcation marked by A occurs. We observe quasiperiodic rotations for the longer delay time $\tau$. Fig. 5(a) shows the quasiperiodic rotation of $\theta(t)$ and control input $u(t)$ at $\tau=1 / 2.1 \mathrm{~s}$.

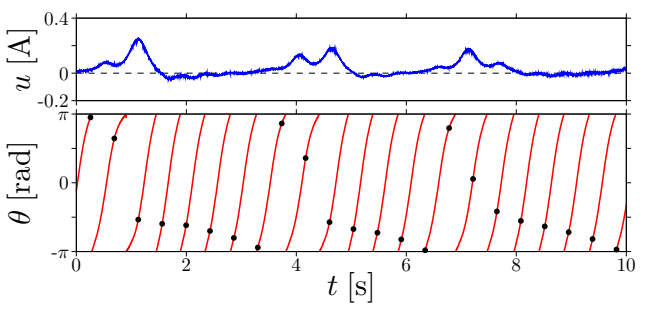

(a) $\tau=1 / 2.1 \mathrm{~s}$.

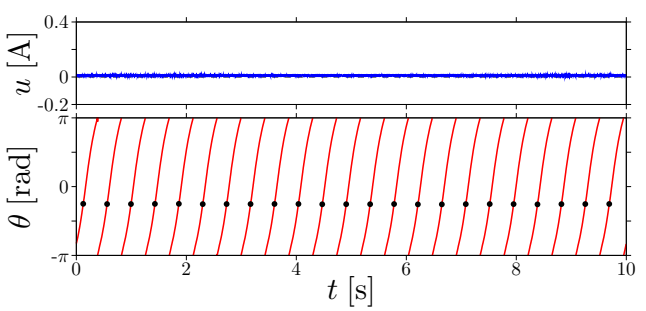

(b) $\tau=T=1 / f=1 / 2.3 \mathrm{~s}$.

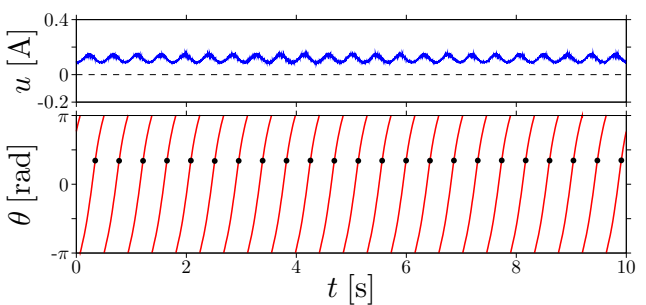

(c) $\tau=1 / 3.0 \mathrm{~s}$

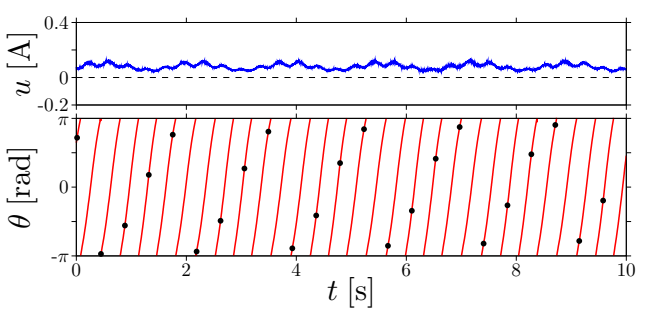

(d) $\tau=1 / 3.4 \mathrm{~s}$.

Figure 5: Steady rotation under the start-up control at the different delay time $\tau$ in the bifurcation diagram of Fig. 4.

The bifurcation diagram elucidates the window of periodic rotation in the domain of the delay. That is, the delayed feedback loop can track a periodic rotation in a certain range of the delay time $\tau$. The existence of the window of periodic rotation in the domain of delay represents the tolerance of proposed control with mistuned delay. The width of window corresponds to the tolerable range of incorrect delay.

For the window of periodic rotation the maximum of input torque is much smaller than the maximum torque induced by the gravity. The periodic rotation can be sustained by the small torque, that is, it implies the sufficiently low energy consumption of the control.

\section{Theoretical discussion on existence of window}

The experimental bifurcation diagram clarified the tolerance of proposed control with mistuned delay. The tolerable range of 


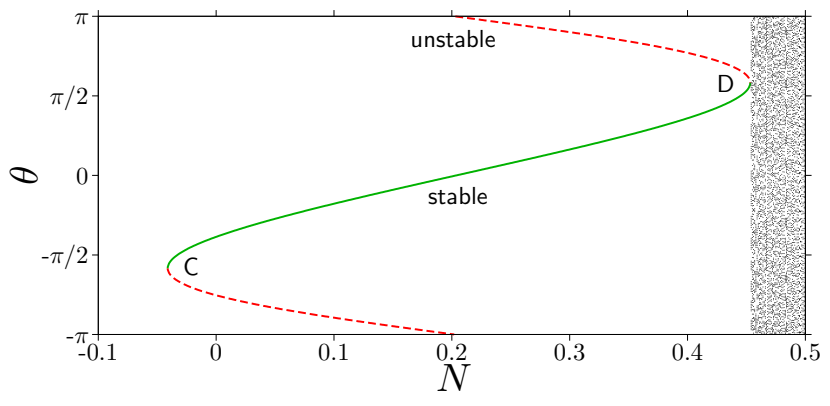

Figure 6: Bifurcation diagram of rotation with respect to the torque $N$ in the driven parametric pendulum (4) at $\gamma=0.1, p=0.5$, and $\omega=2$. The branches consist of the stroboscopic points taken at every excitation period $2 \pi / \omega$.

delay indicates the performance of the start-up control. However, the analysis of the system with delay is complicated because of the infinite dimension of state space. The periodic state represented by the window implies that the infinite dimensional state space degenerates to low dimension. We can theoretically estimate the tolerable range of delay by considering a system without delay.

A system without delay is introduced to model the periodic rotation for the incorrect delay time $\tau$. In the window of the experimental bifurcation diagram the angular displacement $\theta^{*}(t)$ of the periodic rotation can be expressed with a periodic function $x(t)$ :

$$
\theta^{*}(t)=2 \pi f t+x(t), \quad \text { where } \quad x(t)=x(t-T) .
$$

The corresponding control input $u^{*}(t)$ also possesses the same periodicity and is transformed into

$$
u^{*}(t)=K\left(\theta^{*}(t-\tau)+2 \pi-\theta^{*}(t)\right)=2 \pi K(1-f \tau)+y(t),
$$

where $y(t):=K[x(t-\tau)-x(t)]$. The function $y(t)$ obviously has the same periodicity as $x(t)$. From Eq. (3) we simplify the controlled system as the following nondimensionalized parametric pendulum driven by a constant torque $N$ :

$$
\frac{\mathrm{d} \theta}{\mathrm{d} t}=v, \quad \frac{\mathrm{d} v}{\mathrm{~d} t}=-\gamma v-(1+p \cos \omega t) \sin \theta+N
$$

The constant torque $N$ corresponds to $2 \pi K(1-f \tau)$. The linear viscous damping is assumed at the coefficient $\gamma$. We can neglect the periodic input $y(t)$ in the representation because the torque $y(t)$ is sufficiently small in comparison with the effect of the vertical excitation.

Figure 6 shows a bifurcation diagram of rotation with respect to the torque $N$ in the system (4) at $\gamma=0.1, p=0.5$, and $\omega=2$. The stable branch exhibits qualitatively similar structure with the window in the domain of delay in Fig. 4. The stable periodic rotation disappears through the saddle-node bifurcation, denoted by $\mathrm{C}$ and $\mathrm{D}$, at both sides of the branch. For appropriate torque $N$ a stable rotational limit cycle exists in the system (4) without the parametric excitation. The Melnikov's method [20] gives the existence condition $N>4 \gamma / \pi=: N_{0} \approx 0.127$. Thus the rotational limit cycle is entrained by the parametric excitation for $N>N_{0}$. The stable rotation for $N>N_{0}$ corresponds to the entrained rotation. The quasiperiodic rotation through the bifurcation $D$ can be regarded as out of the synchronized state $[21,22]$. Therefore, the bifurcation structure of periodic rotation is governed by the frequency synchronization.

We can qualitatively identify the bifurcations $A$ and $B$ in the experimental setup with the modeled bifurcations $C$ and $D$, respectively. Decreasing (Increasing) delay induces the increase (decrease) of the constant input $2 \pi K(1-f \tau)$ and the change of periodic input $y(t)$, respectively. Here the periodic input $y(t)$ is much smaller than the effect of periodic excitation. Thus, the bifurcation with respect to the delay time $\tau$ in Fig. 4 is caused by the change of the constant input torque $2 \pi K(1-f \tau)$. The bifurcations in Figs. 4 and 6 are accompanied by the change of the constant torque. It is confirmed that the qualitative identification of the bifurcations $A$ and $B$ with the saddle-node bifurcation $C$ and $D$ in terms of the bifurcation with respect to constant torque. The identification implies that the stable branch in the system (4) without delay models the window of periodic rotation in the system (1) with delay. The width of window corresponds to the tolerable range of mistuned delay in the proposed control. Therefore we can estimate the tolerable range of proposed control without analyzing the system with delay in the functional space.

The existence and width of stable branch is governed by the frequency synchronization. Because the system (4) models the system (1) to the extent of the periodic rotation, the existence and width of window of periodic rotation is also governed by the frequency synchronization.

\section{Concluding remarks}

In this paper the window of periodic rotation was experimentally investigated in the bifurcation diagram with respect to delay time under the delayed feedback. We proposed a control method for maintaining the periodic rotation in the vertically excited mechanical pendulum based on the delayed feedback control. The experiments elucidated the periodic window in the domain of delay with small input torque. The existence of the window in the domain of delay represents the tolerance of proposed control with mistuned delay. Therefore the proposed control can maintain the periodic rotation in a certain range of delay with sufficiently low energy consumption. In addition, we introduced a simple model without delay for the periodic rotation of the mechanical pendulum controlled by the delayed feedback. It was confirmed that forced synchronization governed the existence and width of the window. The result implies that the frequency synchronization overcomes the mistuned difference of delay in the control method.

\section{Acknowledgement}

This research was partially supported by the Global COE program of Kyoto University founded by the Ministry of Education, Culture, Sports and Technology of Japan. 


\section{References}

[1] B. P. Koch, R. W. Leven, B. Pompe, C. Wilke, Phys. Lett. A 96 (1983) 219.

[2] R. W. Leven, B. Pompe, C. Wilke, B. P. Koch, Physica D 16 (1995) 371.

[3] M. J. Clifford, S. R. Bishop, J. Sound Vib. 172 (1994) 572.

[4] Y. Yokoi, T. Hikihara, ISCIE J. (to be published) (in Japanese).

[5] K. Pyragas, Phys. Lett. A 170 (1992) 421.

[6] E. Ott, C. Grebogi, J. A. Yorke, Phys. Rev. Lett. 64 (1990) 1196.

[7] H. G. Schuster (Ed.), Handbook of chaos control, Wiley-VCH, Weinheim, 1999.

[8] E. Schöll, H. G. Schuster (Eds.), Handbook of chaos control, 2nd Edition, Wiley-VCH, Weinheim, 2008.

[9] K. Pyragas, A. Tamaševičius, Phys. Lett. A 180 (1993) 99.

[10] S. Bielawski, D. Derozier, P. Glorieux, Phys. Rev. E 49 (1994) R971.

[11] D. J. Gauthier, D. W. S. H. M. Concannon, J. E. S. Socolar, Phys. Rev. E 50 (1994) 2343.

[12] T. Hikihara, T. Kawagoshi, Phys. Lett. A 211 (1996) 29.

[13] T. Hikihara, M. Touno, T. Kawagoshi, Int. J. Bifurcat. Chaos 7 (1997) 2837.

[14] A. G. Balanov, N. B. Janson, E. Schöll, Phys. Rev. E 71 (2005) 016222.

[15] T. Hikihara, Y. Ueda, Chaos 9 (1999) 887.

[16] K. Pyragas, Phil. Trans. R. Soc. A 364 (2006) 2309.

[17] L. M. Pecora, T. L. Carroll, Phys. Rev. Lett. 64 (1990) 821; Phys. Rev. A 44 (1991) 2374.

[18] X. Xu, M. Wiercigroch, M. P. Cartmell, Chaos Soliton. Fract. 23 (2005) 1537.

[19] S. Chiriacescu, Stability in the Dynamics of Metal Cutting, Elsevier, Amsterdam, 1990.

[20] J. Guckenheimer, P. Holmes, Nonlinear Oscillations, Dynamical Systems, and Bifurcations of Vector Fields, Springer, New York, 1983.

[21] M. L. Cartwright, J. Inst. Electr. Eng. 95 (1948) 88.

[22] C. Hayashi, Nonlinear Oscillations in Physical Systems, McGraw-Hill, New York, 1964.

[23] A. Pikovsky, M. Rosenblum, J. Kurths, Synchronization: A universal concept in nonlinear sciences, Cambridge University Press, Cambridge, 2001 